\title{
Corrigendum to "Saturated fat supplementation interacts with dietary forage NDF content during the immediate postpartum period in Holstein cows: Energy balance and metabolism" (J. Dairy Sci. 98:3323-3334)
}

\section{P. Piantoni, A. L. Lock, and M. S. Allen}

On page 3323, the sixth sentence of the abstract should be modified to "Low fNDF diets increased plasma insulin $(0.308$ vs. $0.137 \mu \mathrm{g} / \mathrm{L})$ and glucose concentrations (50.5 vs. $45.7 \mathrm{mg} / \mathrm{dL})$ and decreased plasma nonesterified FA (606 vs. $917 \mu \mathrm{Eq} / \mathrm{L})$ and $\beta$-hydroxybutyrate $(9.29$ vs. $16.5 \mathrm{mg} / \mathrm{dL})$ concentrations and liver triglyceride content."

The first and third rows of Table 2 (page 3329) should be modified as shown below.

The authors regret the errors.

Table 2. Effects of dietary forage NDF (fNDF) content and a saturated free FA supplement (SFFA) on plasma metabolites and hormones of dairy cows during the postpartum period ( 1 to 29 postpartum; $\mathrm{n}=48$ ).

\begin{tabular}{|c|c|c|c|c|c|c|c|c|c|c|c|}
\hline \multirow[b]{2}{*}{ Item } & \multicolumn{2}{|c|}{$20 \% \mathrm{fNDF}$} & \multicolumn{2}{|c|}{$26 \% \mathrm{fNDF}$} & \multirow[b]{2}{*}{ SEM } & \multicolumn{6}{|c|}{$P$-value } \\
\hline & $\begin{array}{c}0 \% \\
\text { SFFA }\end{array}$ & $\begin{array}{c}2 \% \\
\text { SFFA }\end{array}$ & $\begin{array}{c}0 \% \\
\text { SFFA }\end{array}$ & $\begin{array}{c}2 \% \\
\text { SFFA }\end{array}$ & & fNDF & SFFA & $\begin{aligned} & \mathrm{fNDF} \\
& \times \mathrm{SFFA}\end{aligned}$ & $\begin{array}{l}\mathrm{fNDF} \\
\times \text { time }\end{array}$ & $\begin{array}{l}\text { SFFA } \\
\times \text { time }\end{array}$ & $\begin{array}{c}\mathrm{fNDF} \\
\times \mathrm{SFFA} \\
\times \text { time }\end{array}$ \\
\hline Insulin, $\mu \mathrm{g} / \mathbf{L}$ & 0.262 & 0.353 & 0.129 & 0.145 & 0.046 & $<0.001$ & 0.25 & 0.42 & 0.32 & 0.31 & 0.34 \\
\hline Glucagon, $\mathrm{pg} / \mathrm{mL}$ & 118 & 126 & 114 & 120 & 4.91 & 0.30 & 0.13 & 0.88 & 0.01 & 0.41 & 0.16 \\
\hline $\begin{array}{l}\text { Insulin:glucagon, } \\
\text { mol:mol }\end{array}$ & 1.33 & 1.72 & 0.683 & 0.752 & 0.206 & $<0.001$ & 0.28 & 0.45 & 0.53 & 0.28 & 0.30 \\
\hline NEFA, $\mu \mathrm{Eq} / \mathrm{L}$ & 689 & 522 & 965 & 868 & 74.0 & $<0.0001$ & 0.06 & 0.61 & 0.66 & 0.05 & 0.18 \\
\hline BHBA, mg/dL & 8.95 & 9.62 & 15.0 & 17.9 & 1.99 & $<0.001$ & 0.32 & 0.53 & 0.16 & 0.16 & 0.48 \\
\hline $\mathrm{TG}, \mathrm{mg} / \mathrm{dL}$ & 5.81 & 7.19 & 5.22 & 6.33 & 0.421 & 0.06 & $<0.01$ & 0.72 & 0.55 & 0.05 & 0.73 \\
\hline
\end{tabular}

\section{REFERENCES}

Piantoni, P., A. L. Lock, and M. S. Allen. 2015. Saturated fat supplementation interacts with dietary forage NDF content during the immediate postpartum period in Holstein cows: Energy balance and metabolism. J. Dairy Sci. 98(5):3323-3334. https://doi.org/10.3168/jds.2014-8799. 